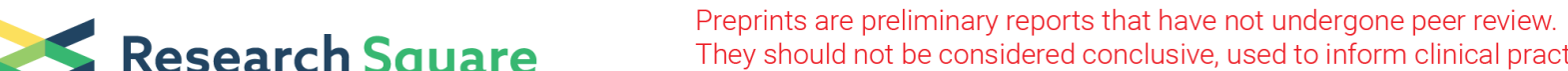 They should not be considered conclusive, used to inform clinical practice, or referenced by the media as validated information.
}

\section{Mechanism of Overcoming Oxaliplatin Resistance Using HT29 Subcloning}

\section{Jisu Lee}

Chungnam National University College of medicine

MI YOON KIM

Chungnam National University College of medicine

\section{Woomi Yang}

Chungnam National University College of Medicine

\section{Il-woung Kim}

Chungnam National University College of Medicine

\section{Seung-Kiel Park}

Chungnam National University College of Medicine Jong-II Park ( $\nabla_{\text {jipark@cnu.ac.kr) }}$

Chungnam National University College of Medicine https://orcid.org/0000-0003-3095-3323

\section{Research article}

Keywords: Colorectal cancer, Heterogeneity, ABCC2, Oxaliplatin, HT29 subcloning

Posted Date: January 9th, 2020

DOl: https://doi.org/10.21203/rs.2.20514/v1

License: (c) (1) This work is licensed under a Creative Commons Attribution 4.0 International License. Read Full License 


\section{Abstract}

Background: Cancer cells are different from normal healthy cells even though they reside within the same tissue and adapt to others. This intra-tumoral heterogeneity is the reason for chemo-resistance, and the emergence of one or more clones, which are resistant to chemotherapeutic drugs, causing clonal outgrowth to form recurrent cancer mass. The need for personalized chemotherapy is increasing because of the tumor heterogeneity and diverse mechanism of chemotherapy resistance. The recent accumulation of extensive evidence for the existence of cancer stem cells strengthens the cancer stem cell hypothesis for chemotherapy resistance and relapse. The development of a primary culture of cancer cells is essential for functional analysis like the sensitivity of chemotherapeutic drugs and the evaluation of the characteristics of cancer stem cells.

Methods: We used a clonal cylinder to establish sub-clones originated from a single cell. Twenty-two subclones were successfully established, and eleven clones were selected according to their growth rate and analyzed. The sub-clones with low expression of BRAF, MEK2, and ERK, but not EGFR or KRAS, showed a correlation with the doubling time. We grouped the sub-clones as the fast-growing group and the slowgrowing group of the HT29 cell line. Three out of five slow-growing sub-clones showed resistance to oxaliplatin treatment. All oxaliplatin-resistant sub-clones overexpressed $A B C C 2$, and no relevance was found with $A B C B 1$ and $A B C G 2$. Active efflux of the drug by $A B C C 2$, but not by $A B C B 1$ or $A B C G 2$, was confirmed with the inhibitor study using each specific inhibitor. The viability of resistant sub-clones decreased after the MK571 treatment, but other clones were not responsive. CD44 expression in oxaliplatin-resistant sub-clones was higher than that of sensitive clones.

Conclusions: This study provides definite evidence of heterogeneity using a cancer cell line. Based on our studies, it appears that intra-tumoral heterogeneity of human cancer tissue is responsible for the development of chemotherapy resistance in cancer. These sub-clones are an excellent model for testing efficacy of anti-cancer drug candidates for advanced cancer therapy. The experimental design and concept of this study could be applied to personalized chemotherapy.

\section{Background}

According to the WCU reports, colorectal cancer (CRC) is the third leading cancer-related cause of death worldwide, and each year it is responsible for 14,000 million deaths [1]. The incidence of colorectal cancer is increasing rapidly in Asian countries because of environmental changes such as increasing average life span and western-style diet [2].

Despite receiving surgical resection and chemotherapy, $50 \%$ of the patients develop tumor relapse and metastasis [3-7]. Surgical therapy is usually applied to a colorectal cancer patient despite the advanced stage at diagnosis, the recurrence rate of which is 20 to $30 \%$ in stage $\nabla$ and 40 to $50 \%$ in stage $\nabla$. Various chemotherapeutic strategies are applied to prevent recurrence, but the development of resistance is the main reason for treatment failure. 
Tumor heterogeneity is defined as the simultaneous presence of multiple clonal sub-populations of tumor cells within a single neoplasm, having different properties including cellular morphology, motility, proliferation, differentiation, metastasis formation, and sensitivity to radiation $[8,9]$. The various cells within a tumor may present significant differences in functional capabilities, such as drug resistance, resistance to radiotherapy and growth rate $[10,11]$. The pathological diagnosis indicates the type of cancer cells, histological grade, and genetic abnormalities. However, present diagnostic routines showed some limitations on the assessment of functional characteristics of heterogeneous cancer cells $[12,13]$.

Recent studies suggest that cancer stem cells, which have self-renewal ability along with multi-lineage differentiation potential and tumorigenic ability, are resistant to chemotherapy and radiotherapy, implying that they are the origin of recurrent cancer [14]. One particularly intriguing property of stem cells is that they express high levels of drug transporters [15] such as ATP-binding cassette (ABC) transporters, which can transport xenobiotic toxins, including anti-cancer drugs. The high expression of $A B C$ transporters is believed to be the main reason for the multi-drug resistance in cancer and cancer stem cells $[16,17]$.

Extensive evidence of intra-tumoral heterogeneity was collected with H\&E stain or immunostaining. However, living cells are needed for the functional study, which can lead to the discovery of new valuable markers. Here, we report definite evidence of cancer heterogeneity in vitro and provide a new system for the functional study of the development of the anti-cancer drug screening system and personalized chemotherapy for relapsed cancer.

\section{Methods}

\section{Cell line and culture conditions}

HT29 human colorectal cancer cell line (ATCC, HTB-38) was maintained in McCoy'5A Medium supplemented with $10 \%$ fetal bovine serum, $1 \%$ Penicillin at $37^{\circ} \mathrm{C}$ in a humidified atmosphere of $5 \% \mathrm{CO} 2$ $(\mathrm{v} / \mathrm{v})$. Cell line was subcultured after detachment with trypsin/EDTA. McCoy'5A medium, fetal bovine serum, penicillin and trypsin/EDTA were purchased from Invitrogen.

\section{Chemicals}

The following drugs were studied: Oxaliplatin (BORYUNG Inc. Seoul, Korea). The concentrations of the studied drugs were in the range from 1 to $500 \mu \mathrm{M}$. The drugs were dissolved in $100 \%$ dimethylsulfoxide (DMSO; Sigma, St. Louis, MO, USA) and then diluted in the media for experiments. In all experiments, control cells were incubated with DMSO alone. The final concentration of DMSO was maintained at $0.2 \%$.

\section{Isolation of sub-populations}

Sub-clones were isolated in parental cell by cloning cylinder (Corning Glass Works, Corning, NY, USA). Distinct colonies are circled with a marker on base $150 \mathrm{~mm}$ dishes. Cloning cylinder is placed with forceps onto the marked circle, into the well of the cloning cylinder trypsinized and transferred in 24-well plates. 


\section{Cell proliferation assay}

Determination of cellular proliferation was accomplished by MTT assay [18]. 4,000 cells/well were seeded in 96-well plates in appropriate medium and incubated at $37^{\circ} \mathrm{C}$ for 6 to $72 \mathrm{~h}$. Medium was removed and wash 3 times with $100 \mu$ of PBS. $100 \mu \mathrm{l}$ of $5 \mathrm{mg} / \mathrm{ml}$ Me3-(4,5-Dimethylthiazolyl)-2,5 diphenyltetrazolium bromide (Sigma, St. Louis, MO, USA) in McCoy medium was added and incubated at $37^{\circ} \mathrm{C}$ for $1 \mathrm{~h}$. Medium was removed and $100 \mu \mathrm{l}$ of DMSO was added to dissolve formazan. The absorbance was measured at $570 \mathrm{~nm}$ in a microplate spectrometer (Thermo Fisher Scientific). All experiments were performed three times. Data plotted are means \pm standard deviation.

\section{Western blot analysis}

Cell were lysed RIPA lysis buffer containing protease inhibitor, cell homogenates containing equivalent amounts of protein were centrifuged at $17000 \mathrm{rpm}$, and the supernatant fractions subjected to SDS-PAGE. Proteins were transferred to polyvinylidene fluoride (PVDF) membranes (Millipore, Billerica, MA). Membranes blocked for $1 \mathrm{~h}$ at room temperature in 5\% skim milk (1\% Tween 20-TBS buffer). Membranes were incubated for $\mathrm{O} / \mathrm{N}$ at $4{ }^{\circ} \mathrm{C}$ with primary antibodies. The antibodies were as follows: EGFR, ERK, $A B C B 1, A B C C 2$ and $A B C G 2$ were obtained from Santa cruz Biotechnology and MEK1, RAS, and RAF were obtained from Cell Signaling Technology. Goat anti-rabbit and anti-mouse secondary antibodies were purchased from Calbiochem. Next, membranes were washed for $1 \mathrm{~h}$ with TBS-T, and incubated with secondary antibody (anti-mouse or anti-rabbit IgG-peroxidase conjugate, 1:2000 dilution in TBS-T) for 1h at room temperature. Membranes were washed for $1 \mathrm{~h}$ with TBS-T. Bound peroxidase was detected using ECL ${ }^{\circledR}$ reagents (Millipore, Billerica, MA, USA).

\section{Cell viability}

4,000 cells of each cell were seeded per well in 96-well plates and incubated overnight. Cells were treated Oxaliplatin (concentration 0.16 to $500 \mu \mathrm{M}$ ). After $72 \mathrm{~h}$, medium was removed and the cells were treated with $200 \mu \mathrm{l}$ of MTT mixture for $2 \mathrm{~h}$ at $37^{\circ} \mathrm{C}$. Subsequently, medium was removed and treated $100 \mu \mathrm{l}$ of Dimethyl sulfoxide. The absorbance was measured at $570 \mathrm{~nm}$ in a microplate spectrometer (Thermo Fisher Scientific). All experiments were performed three times, and data plotted are means \pm standard deviation.

\section{In vivo experiments}

All the animal experiments followed the guidelines of Institutional Review Board (IRB) of animal (No. CNUH-A0012). 5 - 6 weeks old male athymic nude mice were obtained from the Central Lab Animal Inc. (Seoul, Korea) and acclimated for 1 weeks. $2 \times 10^{6}$ cells in $100 \mu$ of PBS were injected subcutaneously in mice right flank and day of the implantation of the tumor cells was designated day $0(\mathrm{DOI} 0) .10 \mathrm{mg} / \mathrm{kg}$ of oxaliplatin or saline was treated intravenously from DI014, once a week, for 30 days. The tumor's greatest 
dimension and the one perpendicular to it were measured every 2 to 3 days using calipers and expressed as length $\mathrm{x}$ width $=$ tumor size and $0.5 \mathrm{x}$ (width) $\mathrm{x}$ length = tumor volume. After the experiment, mice were euthanized with carbon dioxide.

\section{ABC transporter inhibitor studies}

4,000 cells/well of each clones were seeded in 96-well plates and incubated overnight. They were treated with $\mathrm{ABC}$ transporter inhibitor $(20 \mu \mathrm{M}$ of verapamil, $100 \mathrm{nM}$ of mitoxantrone, $40 \mu \mathrm{M}$ of MK571) $1 \mathrm{hr}$ before the treatment of oxaliplatin $(0.16$ to $500 \mu \mathrm{M})$. After $72 \mathrm{~h}$, medium was removed and replaced by $200 \mu \mathrm{l}$ of MTT $(5 \mathrm{mg} / \mathrm{ml})$ in culture media followed by $2 \mathrm{~h}$ incubation $37^{\circ} \mathrm{C}$ incubator. Subsequently, medium was removed and treated $100 \mu \mathrm{l}$ of dimethyl sulfoxide for the solubilization of formazan. The plates were immediately read at $570 \mathrm{~nm}$ using a scanning multi-well spectrometer (Bio-Tek instruments Inc., Winooski, VT). All experiments were performed three times, and data plotted are means \pm standard deviation.

\section{Statistical analysis}

The statistical significance of difference between control and treated groups was analyzed using oneway ANOVA. The difference was considered significant when $p$ value was less than 0.05 and marked as $\star \star \star$ I IC50 values were calculated using the ED50 Plus v1.0 online software.

\section{Results}

\section{Isolation of sub-clones from parent HT29 cells and expression of RAS, RAF and MAPK pathway proteins in HT29 sub-clones}

To investigate the heterogeneity of the cell line, we established sub-clones from HT29 colon cancer cell line. We prepared sub-clones using a traditional cloning cylinder method. The morphology of sub-clones showed a round shape and clustered growth, and almost no difference was observed among clones. However, the growth rate was quite different among sub-clones with a full confluency time ranging from 20 to 32 h (Figure $1 \mathrm{~A})$.

The clone cell stock took 7 to 21 days to prepare. The doubling time was different for each clone. We used western blotting to analyze heterogeneous protein expression in RAS, RAF and MAPK pathway in sub-clones (Figure 1B), which are activated for cell growth, division and differentiation [19]. The expression of EGFR was higher in HT29s01, HT29s02, HT29s06, and HT29s08 than in parental cell line, and the KRAS expression was higher in $\mathrm{HT} 29 \mathrm{~s} 03$ but differed between each clone. The expression of BRAF, MEK, and ERK was lower in HT29s03, HT29s05, HT29s07, and HT29s12 than in other clones.

HT29 cells are EGFR positive, containing wild-type KRAS and mutant BRAF proteins [20]. In practice, it is considered that EGFR-positive as well as KRAS wild-type cells have differences in downstream signaling. The results showed differential expression in the BRAF/MEK/ERK pathway. Six clones (HT29s01, 
HT29s02, HT29s06, HT29s08, HT29s10, HT29s11 cells) exhibited a relatively high level of protein expression than other clones (HT29s03, HT29s05, HT29s07, HT29s09).

\section{The doubling time of each sub-clone}

Distinct subpopulations expressed cell growth pathway proteins, as shown in Figure 1B. We next analyzed the growth rate of eleven clones using the MTT assay. Approximately 4,000 cells/well were seeded in a 96-well plate and allowed to attach for 24,48 , and $72 \mathrm{~h}$ (Figure $1 \mathrm{~A}$ ). The doubling time of parental HT2 29 cells was $23 \mathrm{~h}$, whereas, for each sub-clone, it was different. Growth rates were: $20.3 \mathrm{~h}$ for HT29s01 cells; $20.9 \mathrm{~h}$ for HT29s02 cells; $29.6 \mathrm{~h}$ for HT29s03 cells; $26.25 \mathrm{~h}$ for HT29s0 4 cells; $31.8 \mathrm{~h}$ for HT29s05 cells; $20.9 \mathrm{~h}$ for HT29s06 cells; $28.6 \mathrm{~h}$ for HT29s07 cells; $19.3 \mathrm{~h}$ for HT29s08 cells; $24 \mathrm{~h}$ for HT29s09 cells; $21.1 \mathrm{~h}$ for HT29s10 cells; $21.1 \mathrm{~h}$ for HT29s 11 cells [27]. Heterogeneous growth of the cells in each clone was observed. We divided the cells into two groups according to the doubling time: Fastgrowing group (HT29s01, HT29s02, HT29s08, HT29s10, HT29s11(red lines)), and slow-growing group (HT29s03, HT29s04, HT29s05, HT29s07 (blue lines)).

\section{Oxaliplatin Cytotoxicity in HT29 sub-clones}

Cancer stem cells exhibited a decrease in cellular proliferation. Cells were treated with various concentrations of oxaliplatin for $72 \mathrm{~h}$ to investigate oxaliplatin cytotoxicity in HT29 sub-clones (Figure 2). Oxaliplatin had a different effect on each HT29 sub-clone. HT29 parental cells had an IC50 value of 0.53 $\mu \mathrm{M}$. HT29s01 (IC50 $0.12 \mu \mathrm{M}$ ), HT29s02 (IC50 $0.15 \mu \mathrm{M}$ ), HT29s04 (IC50 $0.58 \mu \mathrm{M})$, HT29s06 (IC50 0.11 $\mu \mathrm{M}), \mathrm{HT} 29 \mathrm{~s} 08(\mathrm{IC} 500.12 \mu \mathrm{M}), \mathrm{HT} 29 \mathrm{~s} 09(\mathrm{IC} 500.62 \mu \mathrm{M}), \mathrm{HT} 29 \mathrm{~s} 10$ (IC50 $0.13 \mu \mathrm{M})$ and HT29s11 (IC50 $0.16 \mu \mathrm{M}$ ) were more sensitive than HT29s03 (IC50 $4.8 \mu \mathrm{M})$, HT29s05 (IC50 $11.1 \mu \mathrm{M}$ ) and HT29s07 (IC50 $3.69 \mu \mathrm{M})$. Parental cells and the fast-growing group were sensitive to oxaliplatin. However, HT29s03, $\mathrm{HT} 29 \mathrm{~s} 05$ and $\mathrm{HT} 29 \mathrm{~s} 07$ cells were resistant to oxaliplatin after $72 \mathrm{~h}$ of exposure. In the previous data (Figure 1A), the slow-growing group had significantly higher chemoresistance than the fast-growing group. Oxaliplatin resistant cells showed the same features as cancer stem cells.

\section{The response of sensitive and resistant cells to oxaliplatin treatment in vivo}

The clones were classified as oxaliplatin sensitive or resistant according to their sensitivity to the oxaliplatin treatment in vitro. However, the sensitivity of cancer cells is not always correlated with in vivo experiments. Therefore, we examined the in vivo response of oxaliplatin in nude mice. The clones, which had similar growth rates, were selected from the oxaliplatin-sensitive (HT29s04) and -resistant group (HT29s05). Further, $2 \times 10^{6}$ cells were subcutaneously inoculated into nude mice flank and oxaliplatin treatment $(10 \mathrm{mg} / \mathrm{kg}$, once a week, for 30 days) followed 2 weeks later. The average final tumor volume of HT29s 04 was $164 \pm 148.6 \mathrm{~mm}^{3}$ in the control group and $46.2 \pm 12.8 \mathrm{~mm}^{3}$ in the oxaliplatin treatment group. Furthermore, the average final tumor volume of HT29s 05 was $442.7 \pm 324.7 \mathrm{~mm}^{3}$ in the control

group, and $385.1 \pm 123.4 \mathrm{~mm}^{3}$ in the oxaliplatin treated group (Figure 3). The treatment of HT29s05 with oxaliplatin did not show a significant difference $(p>0.05)$ in tumor growth, but a dramatic decrease of 
tumor growth was observed in HT29s04, which was in the oxaliplatin sensitive group. These data are consistent with the results of the in vitro cytotoxicity assay.

\section{Expression of $A B C$ transporter in $\mathrm{HT} 29$ sub-clones}

Drug transporters are an important mechanism of chemotherapy resistance. The ABC drug transporters were shown to protect cancer stem cells from chemotherapeutic agents[21]. We examined the protein levels of $A B C$ transporters in the sub-clones using by western blot analysis (Figure 4).

The overexpression of $A B C$ transporters in cancer is considered to be a primary determinant of the multidrug-resistant (MDR) phenotype. We detected the expressions of three primary $A B C$ transporters (ABCB1, ABCC2, ABCG2) by western blot analysis to find whether the resistant cells showed drug resistance to oxaliplatin through these $A B C$ transporters. $A B C G 2$ showed low expression, whereas $A B C B 1$ showed mostly high expression. However, ABCC2 was expressed in high levels in HT29s03, HT29s05, HT29s07 and HT29s09 cells, but not in HT29s04 cells, among the slow-growing group when compared to the fast-growing group.

\section{Discussion}

A variety of cell separation methods are currently available. Fluorescence-activated cell sorting (FACS) is the most popular method due to its convenience and simple procedure. Despite the benefits, FACS has some limitations such as the reliance on the cell surface, limitation in single-cell preparation and a limited number of fractionations for one run [22]. Similarly, magnetic-activated cell sorting (MACS) system is a method for separating various cells using magnetic bead fluorescent antibody. However, purity is the main problem with the MACS system. Therefore, we used the traditional clonal cylinder to establish subclones of the HT29 cell line.

A total of 22 clones were obtained from HT29, but only 11 sub-clones were subjected to analysis, and it is believed to be enough to prove the concept that each clone from one cell line could be heterogeneous at the molecular and functional level. Especially heterogeneity in the expression of growth-related factors, growth rate and sensitivity to oxaliplatin treatment were evident (Fig. 1-2). It is generally believed that slow-growing cells are resistant to anti-cancer drugs $[18,19,22]$. Chen et al. claim that slow-cycling cancer cell populations are resistant to anti-cancer drug treatment. They showed that only quiescent cell populations remain after temozolomide treatment of genetically engineered mouse glioblastoma model, and those are responsible for the recurrence of cancer, adopting cancer stem cell theory [23].

Our data showed that three out of five clones in the slow-growing group were oxaliplatin resistant; however, $\mathrm{HT} 29 \mathrm{~s} 04$ and $\mathrm{HT} 29 \mathrm{~s} 09$, which were classified as slow-growing, were sensitive to oxaliplatin treatment. Expression patterns of the main actors of growth signaling in HT29s04, slow-growing subclone, was almost the same as the fast-growing subclones that had a high expression of BRAF, MEK2 and ERK. The expression of BRAF, MEK2, and ERK was decreased in HT29s09 like in the oxaliplatin resistant group, but it also showed sensitivity with an IC50 of $620 \mathrm{nM}$ when compared to an IC50 of 
11,000 nM (HT29s05). Thus, no correlations between growth rate and oxaliplatin sensitivity were deduced. HT29s04 and HT29s05 were selected for the xenograft experiment because their doubling times were similar (HT29s04: $26.25 \mathrm{~h}$; HT29s05: $31.8 \mathrm{~h}$ ) but the oxaliplatin sensitivity was quite different (IC50 of HT29s04: $0.62 \mu \mathrm{M} ; \mathrm{HT} 29 \mathrm{~s} 05: 11 \mu \mathrm{M}$ ). The growth rate of HT29s04 was faster than that of HT29s05 in vitro, but in the in vivo experiment showed the opposite, where the tumor size of HT29s05 was greater than that of $\mathrm{HT} 29 \mathrm{~s} 04$. Cancer cells interact with the cells and the diverse components in their environment in vivo, and it affects their growth [24], but the environment in the in vitro cell culture system cannot simulate the real environment. EGRF expression in HT29s04 and HT29s05 were almost the same, but the other cell survival factors were low in HT29s05.

The resistance to Platinum (Pt)-based chemotherapeutic compounds by $A B C$ subfamily $C$ transporter has been reported [25]. Among $A B C$ transporters, $A B C B 1, A B C C 2$ and $A B C G 2$ are the most widely evaluated and known, major transporters. Our data showed that the variation of $A B C C 2$ expression was most prominent than others, and the involvement of $A B C C 2$ was confirmed by inhibitor studies (Fig. 4). In summary, the heterogeneous cells exist in the HT29 cell line, and heterogeneous response to anti-cancer drugs is related to $A B C$ transporters.

With this study, we provide definite evidence for the heterogeneity in the HT29 cell line and the importance of establishing subclones for functional assays like the drug sensitivity assay. Further, this study highlights that the average cytotoxicity data of the heterogeneous cells give us little information on the characteristics of the cells and what should be done next. The set of the experiments in this study can be applied to the cells from human cancer tissues and could be a good system for personalized chemotherapy.

\section{Conclusions}

To summarize, this study provides definite evidence of heterogeneity using a cancer cell line. Based on our studies, it appears that intra-tumoral heterogeneity of human cancer tissue is responsible for the development of chemotherapy resistance in cancer. These sub-clones are an excellent model for testing efficacy of anti-cancer drug candidates for advanced cancer therapy. The experimental design and concept of this study could be applied to personalized chemotherapy(Fig. 5).

\section{Declarations}

\section{Ethics approval and consent to participate}

Not applicable.

\section{Consent for publication}

Not applicable. 
Availability of data and materials

All data generated or analysed during this study are included in this published article.

\section{Competing interests}

The authors have declared no conflict of interest.

\section{Funding}

This research was supported by Basic Science Research Program through the National Research Foundation of Korea(NRF) funded by the Ministry of Education(NRF-2017R1A6A3A11032427).

\section{Authors' contributions}

$\mathrm{JL}$ and MYK participated in concept, design, data collection, data analysis, and data interpretation. WY and IWK participated in concept and data interpretation. SKP and JIP participated in data interpretation and made supervision of the study. All authors have read and approved the final manuscript.

\section{Acknowledgements}

We would like to thank Editage (www.editage.co.kr) for English language editing.

\section{Author details}

${ }^{1}$ Department of Biochemistry, College of Medicine, Chungnam National University, Munhwa-ro 266, Junggu, Daejeon, 35015, South Korea

${ }^{2}$ Cancer Research Institute, Chungnam National University, Munhwa-ro 266, Jung-gu, Daejeon, 35015, South Korea

\section{Abbreviations}

WCU: Western Carolina University; CRC: colorectal cancer; ABC:ATP-binding cassette; MDR: multidrugresistant; FACS: Fluorescence-activated cell sorting; MACS: magnetic-activated cell sorting; PVDF: polyvinylidene fluoride; EGFR: Epidermal growth factor receptor; KRAS: Kirsten RAt Sarcoma; BRAF: v-raf murine sarcoma viral oncogene homolog B1; MEK: Mitogen-activated protein kinase kinase and MAP2K; ERK: Extracellular signal-regulated kinase; MAPKs: Mitogen-activated protein kinases; Pt:Platinum DOI: Day of Injection

\section{References}

1. Shin A, Kim KZ, Jung KW, Park S, Won YJ, Kim J, Kim DY, Oh JH: Increasing trend of colorectal cancer incidence in Korea, 1999-2009. Cancer Res Treat 2012, 44(4):219-26. 
2. MA P: Epidemiology and burden of colorectal cancer in Asia-Pacific region: what shall we do now? Trans/ Gastrointest Cancer 2014, 3(4):169-73.

3. Huh JW, Kim CH, Lim SW, Kim HR, Kim YJ: Early recurrence in patients undergoing curative surgery for colorectal cancer: is it a predictor for poor overall survival? Int J Colorectal Dis 2013, 28(8):11439.

4. Saunders M, Iveson T: Management of advanced colorectal cancer: state of the art.Brit J Cancer 2006, 95(2):131-8.

5. Galandiuk S, Wieand HS, Moertel CG, Cha SS, Fitzgibbons RJ, Jr., Pemberton JH, Wolff BG: Patterns of recurrence after curative resection of carcinoma of the colon and rectum. Surg Gynecol Obstet 1992, 174(1):27-32.

6. Heald RJ, Ryall RD: Recurrence and survival after total mesorectal excision for rectal cancer.Lancet 1986, 1(8496):1479-82.

7. Stipa S, Nicolanti V, Botti C, Cosimelli M, Mannella E, Stipa F, Giannarelli D, Bangrazi C, Cavaliere R: Local recurrence after curative resection for colorectal cancer: frequency, risk factors and treatment. $J$ Surg Oncol Supp/ 1991; 2:155-60.

8. Heppner GH. Tumor heterogeneity.Cancer Res 1984, 44(6):2259-65.

9. Fidler IJ, Hart IR: Biological diversity in metastatic neoplasms: origins and implications. Science 1982, 217(4564):998-1003.

10. Salmon SE, Hamburger AW, Soehnlen B, Durie BG, Alberts DS, Moon TE: Quantitation of differential sensitivity of human-tumor stem cells to anticancer drugs.N Engl J Med 1978, 298(24):1321-7.

11. Heppner GH, Miller BE: Tumor heterogeneity: biological implications and therapeutic consequences.Cancer Metastasis Rev 1983, 2(1):5-23.

12. Sadanandam A, Lyssiotis CA, Homicsko K, Collisson EA, Gibb WJ, Wullschleger S, Ostos LCG, Lannon WA, Grotzinger C, Del Rio M, Lhermitte B, Olshen AB, Wiedenmann B, Cantley LC, Gray JW, Hanahan D: A colorectal cancer classification system that associates cellular phenotype and responses to therapy.Nat Med 2013, 19(5):619-25.

13. Schnitt SJ: Classification and prognosis of invasive breast cancer: from morphology to molecular taxonomy.Mod Pathol 2010, 23 Suppl 2:S60-4.

14. Pardal R, Clarke MF, Morrison SJ: Applying the principles of stem-cell biology to cancer.Nat Rev Cancer 2003, 3(12):895-902.

15. Bose D, Zimmerman LJ, Pierobon M, Petricoin E, Tozzi F, Parikh A, Fan F, Dallas N, Xia L, Gaur P, Samuel S, Liebler DC, Ellis LM: Chemoresistant colorectal cancer cells and cancer stem cells mediate growth and survival of bystander cells.Brit J Cancer 2011, 105(11):1759-67.

16. Cole SPC, Bhardwaj G, Gerlach JH, Mackie JE, Grant CE, Almquist KC, Stewart AJ, Kurz EU, Duncan AMV, Deeley RG: Overexpression of a transporter gene in a multidrug-resistant human lung-cancer cell-line.Science 1992, 258(5088):1650-4. 
17. Hlavata I, Mohelnikova-Duchonova B, Vaclavikova R, Liska V, Pitule P, Novak P, Bruha J, Vycital O, Holubec L, Treska V, Vodicka P, Soucek P: The role of ABC transporters in progression and clinical outcome of colorectal cancer. Mutagenesis 2012, 27(2):187-96.

18. Dallas NA, Xia L, Fan F, Gray MJ, Gaur P, van Buren G, Samuel S, Kim MP, Lim SJ, Ellis LM: Chemoresistant colorectal cancer cells, the cancer stem cell phenotype, and increased sensitivity to insulin-like growth factor-I receptor inhibition. Cancer Research 2009, 69(5):1951-7.

19. Cary LA, Han DC, Guan JL: Integrin-mediated signal transduction pathways. Histol Histopatho/ 1999, 14(3):1001-9.

20. Matsuo T, Nishizuka SS, Ishida K, Iwaya T, Ikeda M, Wakabayashi G: Analysis of the anti-tumor effect of cetuximab using protein kinetics and mouse xenograft models.BMC Res Notes 2011, 4:140.

21. Moitra K, Lou H, Dean M: Multidrug efflux pumps and cancer stem cells: insights into multidrug resistance and therapeutic development. Clin Pharmacol Ther 2011, 89(4):491-502.

22. Tomlinson MJ, Tomlinson S, Yang XB, Kirkham J: Cell separation: Terminology and practical considerations.J Tissue Eng 2013, 4:2041731412472690.

23. Chen J, Li Y, Yu TS, McKay RM, Burns DK, Kernie SG, Parada LF: A restricted cell population propagates glioblastoma growth after chemotherapy. Nature 2012, 488(7412):522-6.

24. Whiteside TL: The tumor microenvironment and its role in promoting tumor growth. Oncogene 2008, 27(45):5904-12.

25. Handgretinger R, Lang P, Schumm M, Taylor G, Neu S, Koscielnak E, Niethammer D, Klingebiel T: Isolation and transplantation of autologous peripheral CD34+ progenitor cells highly purified by magnetic-activated cell sorting.Bone Marrow Transplant 1998, 21(10):987-93.

\section{Figures}


A

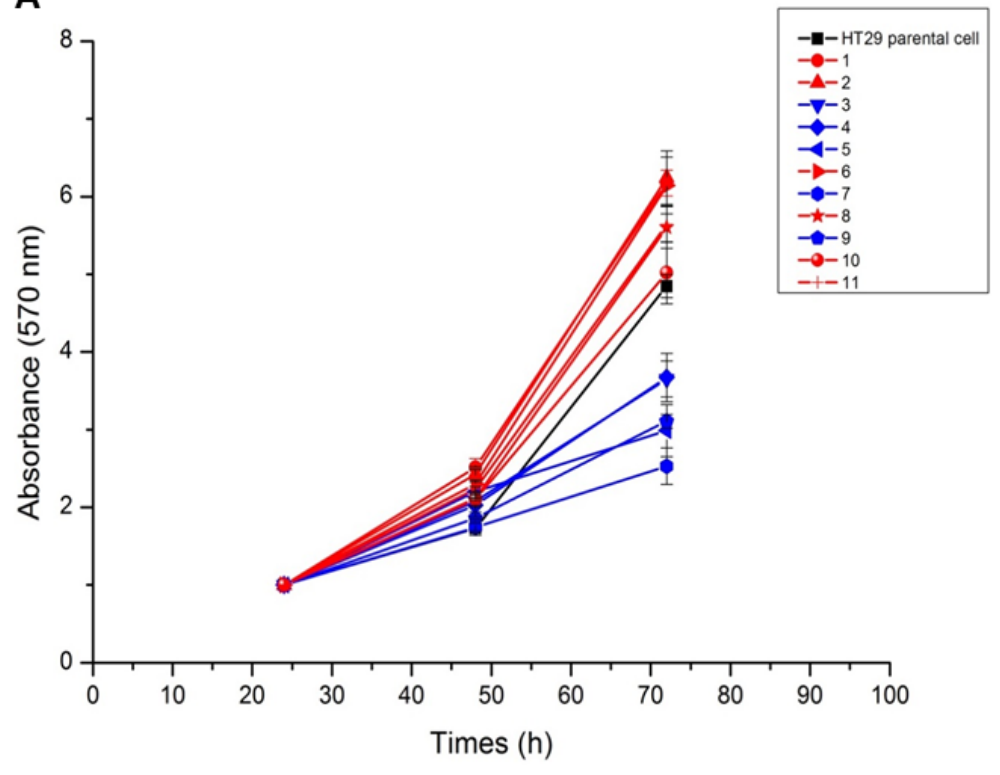

B

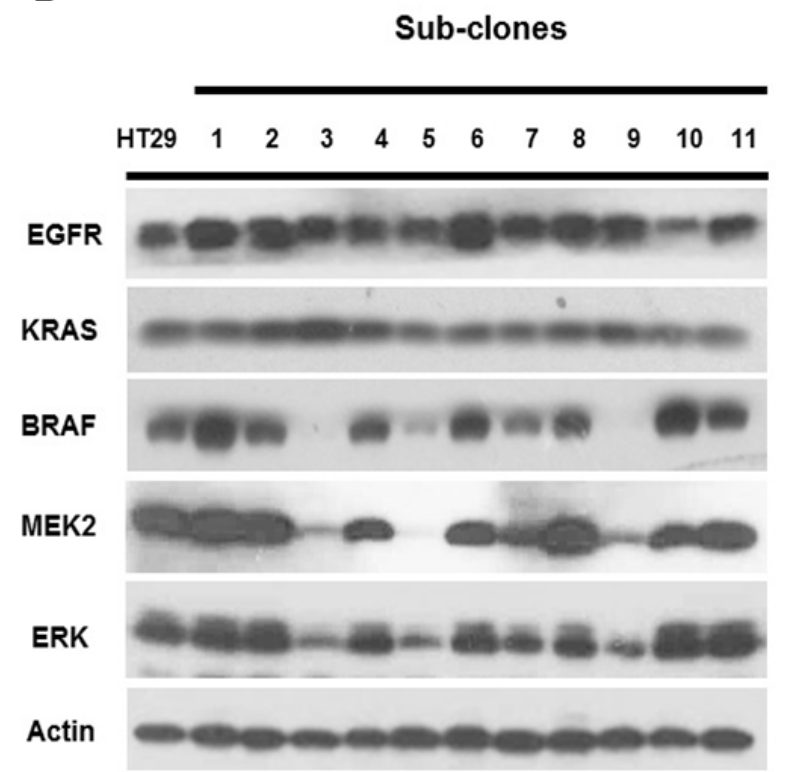

\section{Figure 1}

Growth rate and expression level of MAPK in HT29 subclones. (A) For 24 to $72 \mathrm{~h}$, growth rates were evaluated in MTT assay. Sub-clones were divided two groups to be based on parental cells. Fast-growing group (HT29s01, HT29s02, HT29s08, HT29s10, HT29s11(red lines)), and slow-growing group (HT29s03, HT29s04, HT29s05, HT29s07 (blue lines)). All experiments were performed three times, and data plotted are means $\otimes$ standard deviation. (B) Expression levels of EGFR, RAS, RAF and MAPK of each clone were determined by western blot and actin was used as a loading control. Note, sub-clones were showed heterogeneity in the expression of growth signaling molecules. 


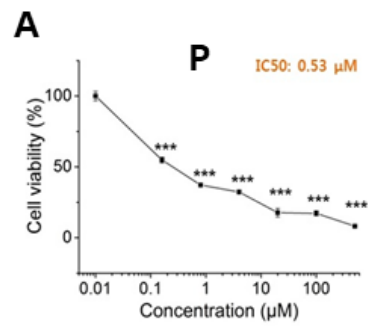

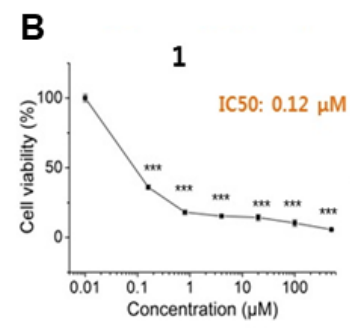

6
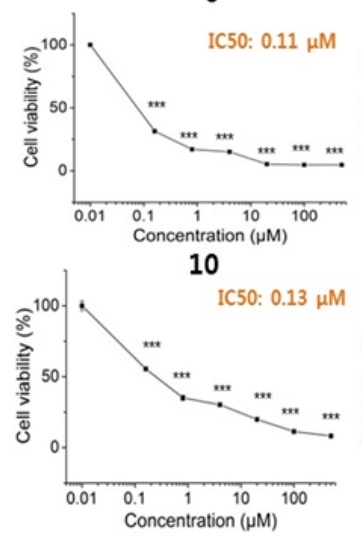
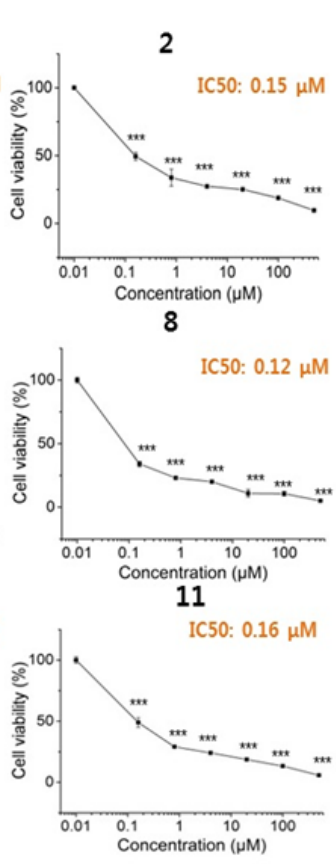

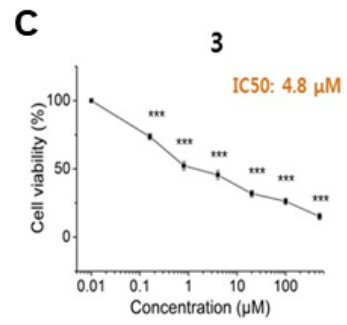

5

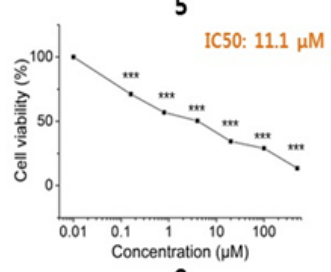

9

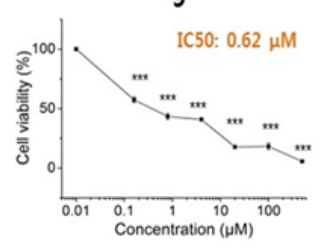

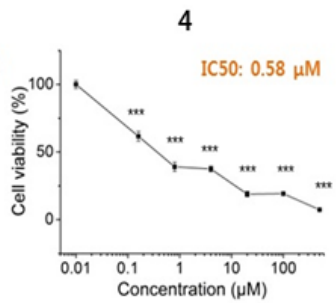

7

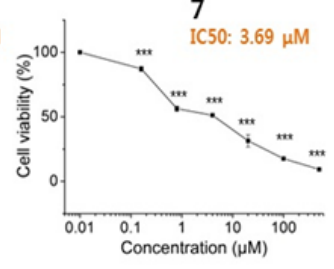

\section{Figure 2}

Cytotoxicity of oxaliplatin in HT29 sub-clones. Sub-clones were seeded in 96well plate. After 24h, HT29 cells were exposed at different concentrations ranging from 0.16 to $500 \mu \mathrm{M}$ of Oxaliplatin for $72 \mathrm{~h}(\mathrm{~A}-\mathrm{C})$. Data represent the mean percentage of cell viability $( \pm S D)$ from three independent experiments. The difference was considered significant when $p$ value was less than 0.05 and marked as $* \star *$. 

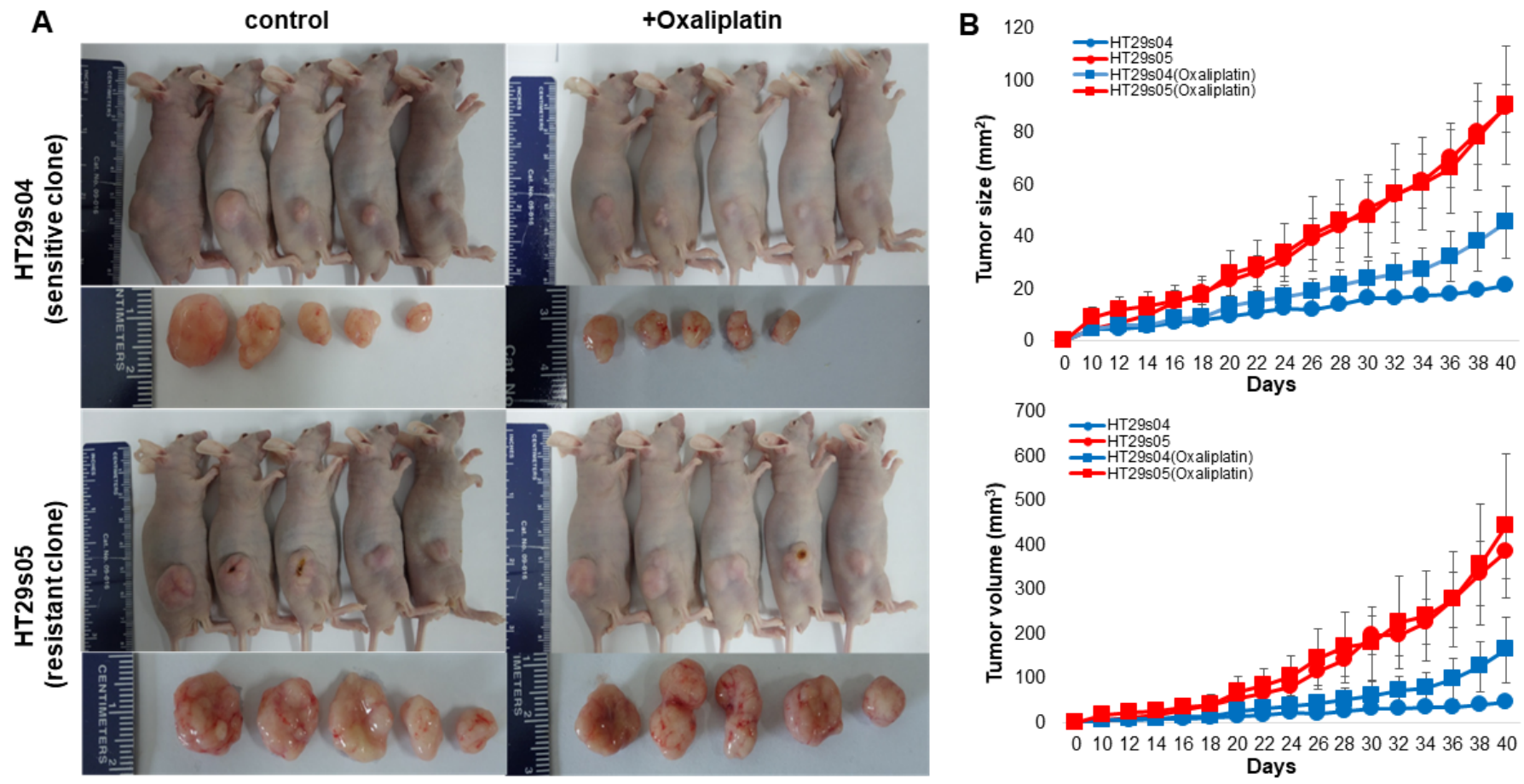

\section{Figure 3}

The response of sensitive and resistant clones to the treatment of oxaliplatin. (A) HT29s04 (sensitive clone) and HT29s05 (resistant clone) were subcutaneously inoculated into nude mice. Two weeks later, mouse was treated with oxaliplatin $10 \mathrm{mg} / \mathrm{kg}$ once a week for 30 days. (B) The data plotted are mean $\pm S D$ of size and volume of tumor. 


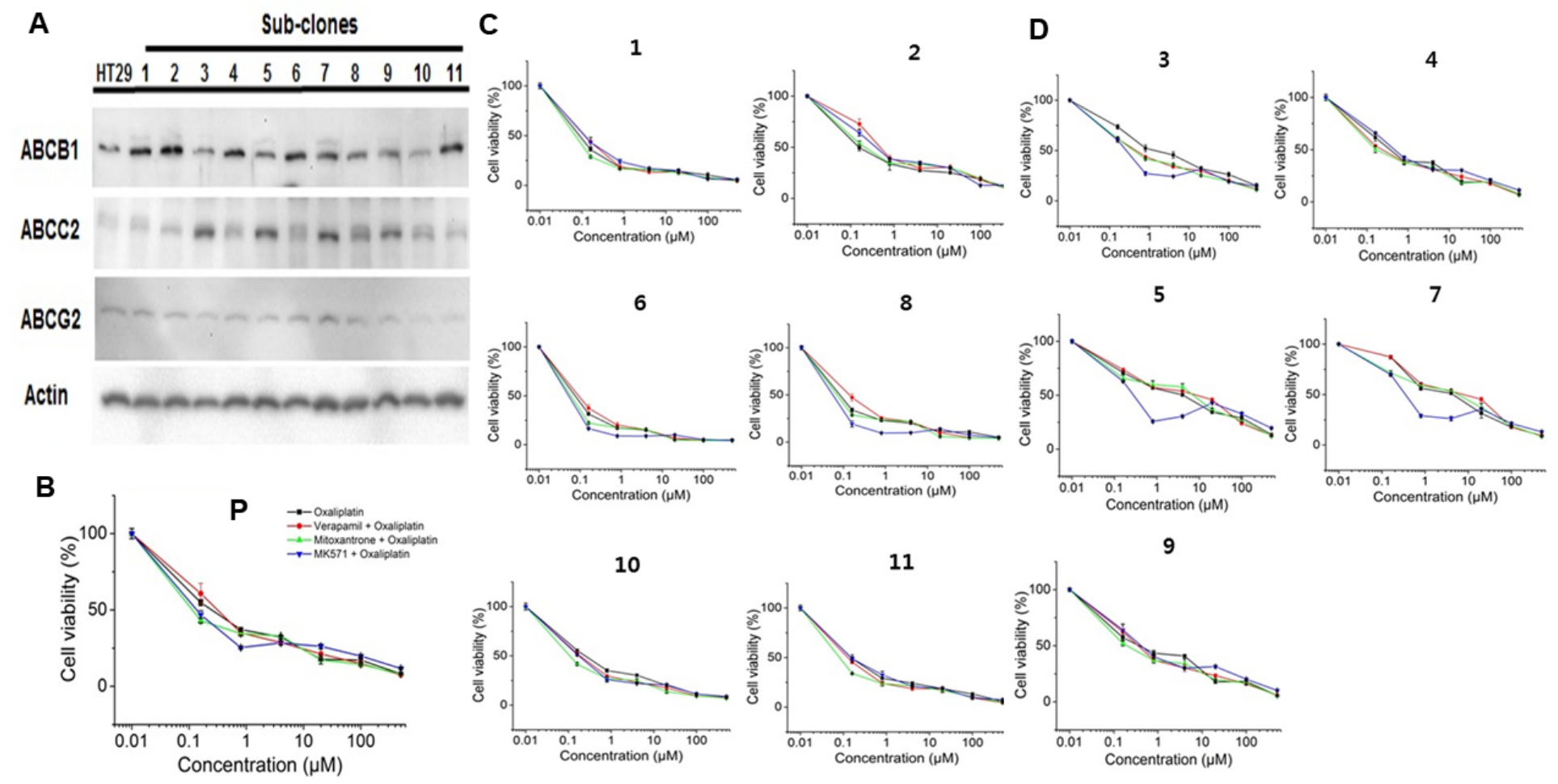

\section{Figure 4}

Oxaliplatin resistant cells caused by high expression of $A B C C 2$. Expression levels of $A B C$ transporter were analyzed by Western blot (A) and inhibition studies was done by MTT assay (B-D). Sub-clones showed heterogeneity in the $A B C C 2$ expression (A). Combinatorial treatment of $A B C$ transporter inhibitor and oxaliplatin showed no difference between drug sensitive group and HT29 parental cells but the cytotoxic effect of oxaliplatin was enhanced by ABCC2 inhibitor in oxaliplatin resistant cells (B-D). 


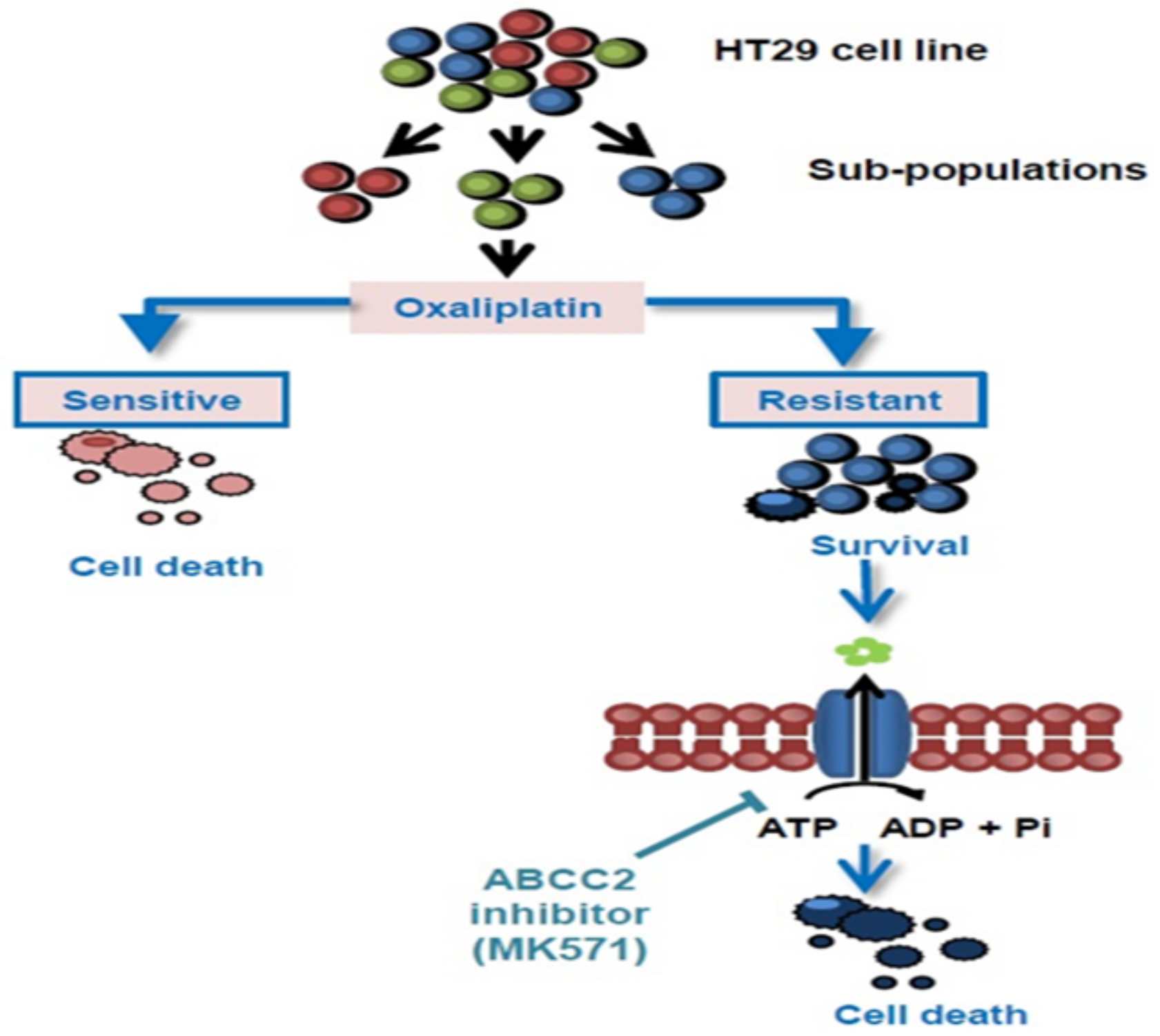

Figure 5

Proposed schematic diagram of functional heterogeneity in HT29 cells.

\section{Supplementary Files}

This is a list of supplementary files associated with this preprint. Click to download.

- NC3RsARRIVEGuidelinesChecklistfillable20191231.pdf 